\title{
On Noisy Fading Channel, Image Transmission Using Orthogonal Frequency Division Multiplexing System
}

\author{
Ghassan Muslim Hassan ${ }^{1 *}$, Mohad Rosmadi Mokhtar ${ }^{2}$, \\ Raed Ali Al-Saqour ${ }^{3}$ and Khairul Azmi Abu Bakar ${ }^{4}$ \\ ${ }^{1}$ College of Science - Computer Dep. -Al-Mustansiriyah University, Baghdad, Iraq \\ ${ }^{2}$ Faculty of Information Science \& Technology, UKM, Bangi, Malaysia \\ ${ }^{3}$ College of Computer and Information, Saudi Electronic University, Jeddah, \\ Kingdom of Saudi Arabia \\ ${ }^{4}$ Faculty of Information Science \& Technology, UKM, Bangi, Malaysia \\ lgmhalsaddi@siswa.ukm.edu.my,'rgmhalsaddi@yahoo.com, \\ 2mrm@ukm.edu.my, ${ }^{3}$ raed.ftsm@gmail.com, ${ }^{4}$ khairul.azmi@ukm.edu.my
}

\begin{abstract}
Given the ability of orthogonal frequency division multiplexing (OFDM) to manage strict channels, it is a largely accepted multicarrier modulation method in current wireless communications, especially those with high data rate. In this study, an image was transmitted with considering the power consumption, which was received by the noisy channel of the OFDM systems. Many factors had been tested to support the work and to make the ideas specifically, the size of the fast Fourier transforms/inverse fast Fourier transform (FFT/IFFT) was tested to minimize complexity, which is the behavior of a system's process of transmitting and receiving data with less suffering. The bandwidth $(B W)$ size was also considered, and who much it effects of the transmission process. The type of modulation, to choose the type of modulation, we use phase shift keying (PSK) and quadrature amplitude modulation (QAM) on the simulation and obtain a perfect modulation type of OFDM systems. Furthermore, signal-to-noise ratio (SNR), which is one of the main performance metrics of OFDM systems, was considered to determine its effect on the signal. Simulation results show, the effect of SNR on the bit error rate (BER) and the effect of the FFT number (nFFT) on the BER. Peak-to-power ratio (PAPR) is the main disadvantage of OFDM, thus our results take into account an effect of the nFFT on PAPR. The study focused on the BER with respect to SNR and to the $n F F T$, as well as the PAPR with respect to the nFFT. All simulation results were defined using a channel of additive white Gaussian noise (AWGN). Performance evaluation results were obtained through simulation using MATLAB.
\end{abstract}

Keywords: OFDM, PAPR, BER, IFFT/FFT, SNR.

\section{Introduction}

In the early 1990s, with the development of hardware, digital signal processing made orthogonal frequency division multiplexing (OFDM) a realistic option for wireless systems [1]. Recently, OFDM emerged as a key candidate for high-data-rate applications. It is now used for digital audio broadcasting, digital video broadcasting, and wireless local area networks (IEEE802.11a, IEEE802.11g). It will also be used in fourth-generation cellular systems and beyond, including third-generation partnership project long-term evolution and WiMAX [2].

The OFDM technique involves the following: "The signal of the radio is split into multiple smaller sub-signals," then "These sub-signals are transmitted to the receiver

Corresponding Author ${ }^{*}$ : gmhalsaddi@siswa.ukm.edu.my, gmhalsaddi@yahoo.com Phone : +601137901806 
simultaneously with different frequencies." Therefore, the main BW is split into multi subbands, and the crosstalk in signal has reduced using OFDM. When an image is transmitted over noisy channels, the data are either missing or incorrect because of the channel transmission errors [3]. However, image transmission over the fading channels without degrading the perceptual quality and while mitigating the power consumption in many fields, such as broadband networks, mobile communications, image sharing, and video broadcasting (VB), is a challenging task. Re-sending the lost packets every time in numerous applications, such as VB [6], is likewise impractical. Several of OFDM's advantages are its facility to deal with severe channel conditions without the need for a complex equalization filter, for example, frequency selective fading, as a result of multipath and narrow band interference. In OFDM, a large number of orthogonal, overlapping, and narrow band subcarriers are transmitted in parallel and used in digital communication [5]. The spectra of these sub-carriers are closely spaced and overlapped to achieve high BW efficiency. The BW of these sub-carriers become small compared with the coherence BW of the channel [4]. OFDM allows digital data, including image data, to be efficient and reliably transmitted in multipath environments by lowering the symbol rate [4]. The algorithm has been implemented to read the stored image (features such as color, shape, and robust) and transmit it by choosing all types of modulation with different number of fast Fourier transform and SNR, which is equal to $20 \mathrm{~dB}$. Changing the modulation type will also change the constellation, which will be helpful in determining the effectiveness of the transmitted data. The method of the channel estimator is the most important technique to estimate the incoming signal. In the present work, least squares (LS) estimators have been chosen in changing the parameters of the estimator to enhance the estimation process of LS.

The paper is organized as follows. Section 2 shows the literature review for OFDM studies, while Section 3 describes a background of OFDM with an important performance, namely, bit error rate (BER). A methodology of the work with send and receive data (image), which was performed by the proposed algorithm, is also in this section. Section 4 explains the peak-to-power ratio (PAPR), which is the main part of the drawback in OFDM. The simulation analysis and results are discussed in Section 5. Finally, the conclusion is presented in Section 6.

\section{Literature Review}

Reference [6] proposes an effective technique for color image transmission with a power saving approach over the OFDM system. The reception quality of the receiving image is also perfect enough with various peak signal-to-noise ratios (PSNR) that save $60 \%$ energy. However, the synchronization problem did not consider including the PAPR problem.

Reference [7] shows that adding discrete sine transform to the system improves the visual quality of reconstructed images and reduces the PAPR of the OFDM signal. The study focuses on greatly reducing the PAPR present in the OFDM signal (causing nonlinearity at the receiving end). The synchronization of the transmitted signal is not considered, and BER is not mentioned. Therefore, a drawback exists in this study.

In [8], the authors present an energy-saving approach to the transmission of discrete wavelet transform (DWT) -based compressed image frames over the OFDM channels. The descriptions in order of descending priority are assigned to the currently perfect channels on the basis of one-bit channel state information at the transmitter. The authors do not introduce anything about the PAPR drawback and the BER performance.

The authors in [9] show an image that is compressed using the DWT, and the compressed data are arranged in four subbands. These subbands are packetized and serially mapped to the OFDM system. After receiving the channels at the receiver, the bad 
channel drops at the receiver side. The main drawbacks of this study are the synchronization and BER.

In [10], the authors describe the Simulink implementation of all functional blocks of a standard digital video broadcasting (DVB)-T transmitter and receiver and presents the simulation model for the DVB-T system according to the European Telecommunications Standards Institute (ETSI EN 300744 V1.6.1). The implemented model contains all channel coding and modulation building blocks with the following parameters: $8 \mathrm{~K}$ OFDM-mode, code rate (1/2, 2/3, and 3/4), and modulation (64-QAM, 16-QAM).

In [11], the authors propose an estimator for the channel delay spread and analyzed the DVB system. The authors consider the synchronization problem and frequency Doppler. PAPR is the main disadvantage of OFDM, but it is not mentioned in the study.

Reference [12] shows an image frame that is compressed using the DWT, and the compressed data are arranged in data vectors, each with an equal number of coefficients. These vectors are quantized and binary coded to obtain the bit streams, which are packetized and intelligently mapped to the OFDM system.

In [13], the PSNR performance of the conventional fast Fourier transforms (FFT) OFDM system and discrete cosine transform (DCT) -OFDM system is compared with the DWT-OFDM system in a Gaussian noise environment along with error correcting codes, such as Reed-Solomon and low-density parity-check.

Authors in [14] discuss their carrier frequency offset estimation algorithms and focus on its performance in influencing the OFDM system with low-precision quantization. Theoretical analysis and simulation figures verify that the quantization error is introduced because of the low-precision quantization, which then significantly degrades the performance of the receiver system.

In [15], the term immunity to impulse interference, high spectral density, robustness to channel fading, multipath, and much lower computational complexity are discussed. All the parameters are compared using additive white Gaussian noise and Rayleigh fading channel by changing the phase of several subcarriers using QPSK in OFDM modulation.

Authors in [16] proposed an enhanced algorithm of the timing synchronization which implemented the training sequence, and the estimation function of the timing synchronization which improved for this algorithm.

\section{Methodology}

\subsection{Background}

OFDM is found to have total immunity to multipath delay spread provided the reflection time is less than the guard period used in the OFDM signal. Figure 1 shows the block diagram of a typical OFDM transceiver (transmitter + receiver) $[17,18]$. 
Tx.

Input Binary Data

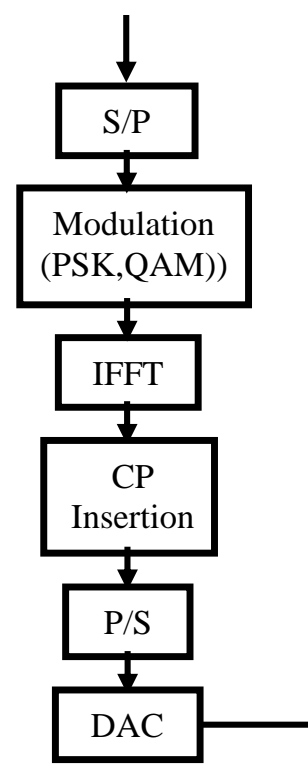

Rx.

Output Binary Data

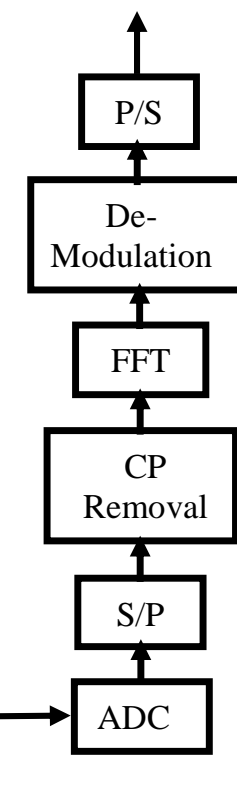

Figure 1. OFDM Generation And Reception Block Diagram

The transmitter section converts binary data for transmission into a mapping of subcarrier amplitude and phase. After converting the input binary data from serial to parallel (S/P) and modulation using phase shift keying (PSK) or quadrature amplitude modulation (QAM), the spectral representation of the data transforms from frequency domain to time domain using an inverse FFT. Furthermore, cyclic prefix (CP) is added to assist in the synchronization. A conversion of the data occurs from parallel to serial $(\mathrm{P} / \mathrm{S})$ followed by conversion from digital to analog. The output signal is as follows:

$x(n)=\frac{1}{\sqrt{N}} \sum_{m=0}^{N-1} x(m) \cdot e^{\frac{j 2 \pi n m}{N}}$

where $x(n)$ is the OFDM signal and $X(m)$ is the modulated symbol in frequency domain for the $m^{\text {th }}$ subcarrier. By contrast, $N-1 \geq m \geq 0, \mathrm{n}$ is a time domain sample index, and $N$ denotes the number of subcarriers.

The incoming signal will be mixed with AWGN in the channel. The receiver performs the reverse operation of the transmitter. Before the FFT, there are analog to digital conversion, $\mathrm{S} / \mathrm{P}$, and $\mathrm{CP}$ removal; FFT also analyzes the signal in the frequency domain. The amplitude and phase of the subcarriers are identified and converted back to digital data

$$
Y(m)=\sum_{n=0}^{N-1} y(n) \cdot e^{\frac{j 2 \pi n \varepsilon}{N}}+w(n)
$$

where $Y(m)$ is the output of the FFT stage, $w(n)$ is the AWGN, $y(n)$ is the received signal after passing through the AWGN channel affected by frequency offset, and $\varepsilon$ is the normalized frequency offset and is given by $\Delta f N T s$, where $\Delta f$ is the frequency difference of the local oscillator between transmitter and receiver, and $T s$ is the symbol period.

An important factor in a communication system is BER, which is a main performance in the receiver side. It may be affected by noise, interference, distortion, and wireless 
multipath fading. The BER may be improved by choosing a strong signal strength, choosing a slower and a robust modulation scheme or line coding scheme, and applying channel coding schemes, such as redundant forward error correction codes [2]. Generally, the BER can be expressed mathematically as follows:

$B E R=\frac{\text { number of errors }}{\text { total number of bits sent }}$

Several factors can affect BER estimation, such as the form of the data, which are sent through a BER measurement that can influence the result. Interference of adjacent channels, fake signals from additional channels, interference of sweep transmitter, inchannel access (burst noise and impulse), and incorrectly aligned and/or faulty amplifiers are several degrading causes of BER.

\subsection{Proposed Scheme}

The algorithm has three phases;

i) Phase 1, transmitter

After modulation type had been chosen for modulation (BPSK, QPSK, 8PSK, 16QAM, 32QAM, AND 64QAM), the processes of reading an image, convert data type to binary and convert from serial to parallel. Inserting cyclic prefix to the data after applying IFFT to convert the domain from frequency to time domain. Finally, the data had been converted again from parallel to serial. The OFDM signal is ready to transmit which is represented by equation 1. Calculation of PAPR is used in the calculation and performance of the algorithm.

ii) Phase 2, channel

Through the transmission channel, AWGN and noise are added to the OFDM signal. SNR takes more than one value $(-5,0,5, \ldots ., 40)$. At this stage, the power of data is calculated for use in our calculations.

iii) Phase 3, receiver

The inverse operations are done, which include converting from serial to parallel, removing $\mathrm{CP}$, converting the data from the time domain to the frequency domain by using FFT, estimating channels, applying demodulation, and converting from parallel to serial which represented as equation 2 . Through these operations, BER has been calculated to see the performance of the algorithm.

\section{Drawback of OFDM}

One of the principal drawbacks of OFDM is PAPR. This vital issue of subcarriers force vacillation forces that have the undesirable multifaceted nature of digital to analog conversion, as they should work in a more extensive element range. A power amplification, which is situated on the transmitter part, should also work in an enormous straight area to avoid spectral development. Therefore, noise becomes out-of-band. All past requirements are important for the presence of high PAPR, which builds the general expense of an OFDM system.

In a system with multi-carrier, if the subcarriers are out of phase between each other, then PAPR will happen. PAPR is relative between the maximum sample power value in a transmitted OFDM signal, divided by an average power of the same OFDM signal in equation 4. 
PAPR $=\frac{\text { Ppeak }}{\text { Paverage }}=\frac{\max \left[|X(n)|^{2}\right]}{E\left[|X(n)|^{2}\right]}$

where $P_{\text {peak }}$ is the peak of power output, $P_{\text {average }}$ is the average of power, $E[]$ is the expected value, and $X(n)$ is the transmitted signal of OFDM.

Reducing the power of the transmitted signal to bypass the effect of PAPR will, therefore, reduce the SNR and then to reduce BER. So, there are many techniques which are used or reduction PAPR [19-23].

\section{Simulation Results}

Figure 2, illustrates when the algorithm has been implemented,. Each run of the algorithm by choosing the modulation type and fixing the value of SNR, number of FFT samples was changed which is lead to calculation BER. Table 1 shows the summary of the results

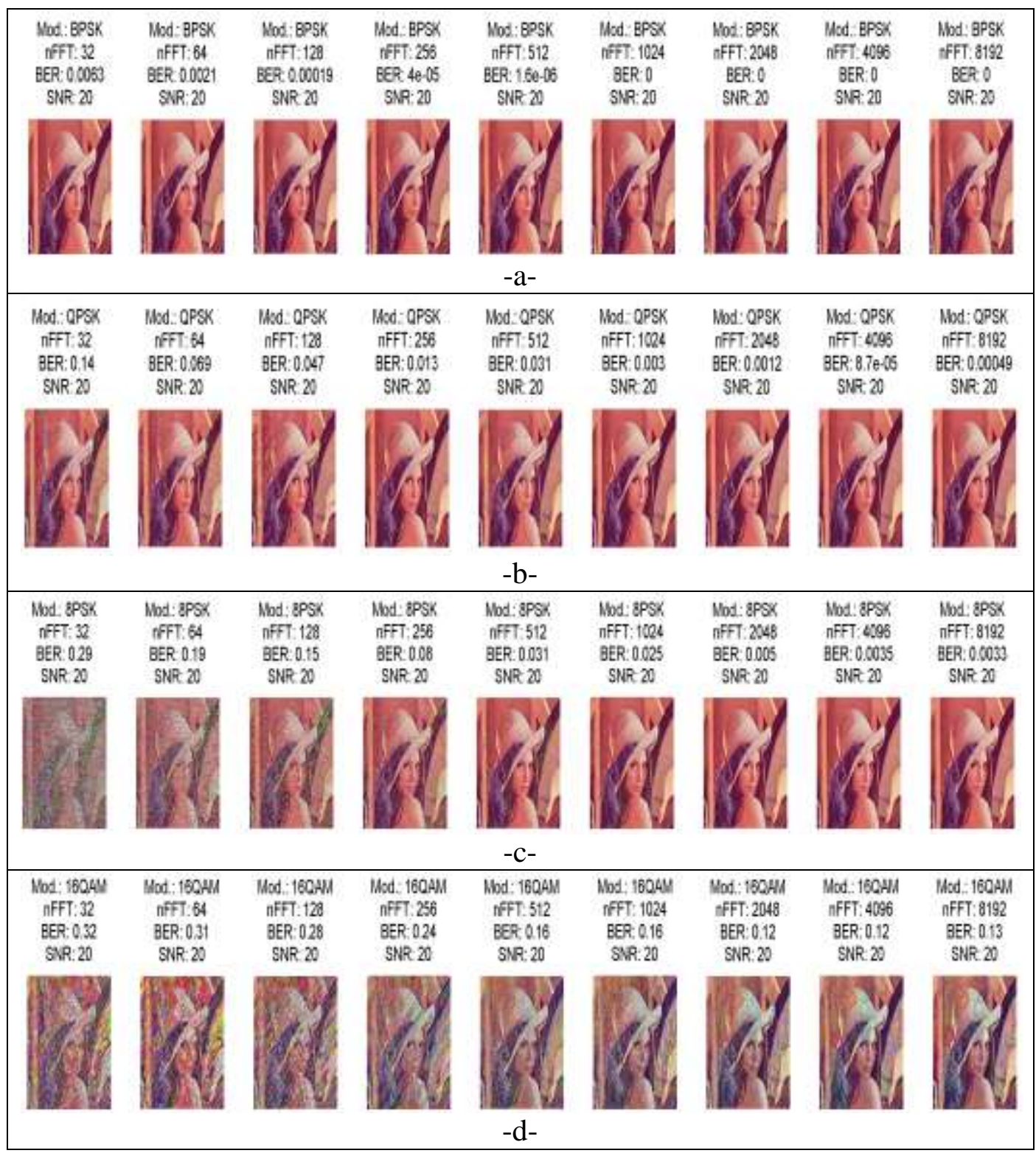




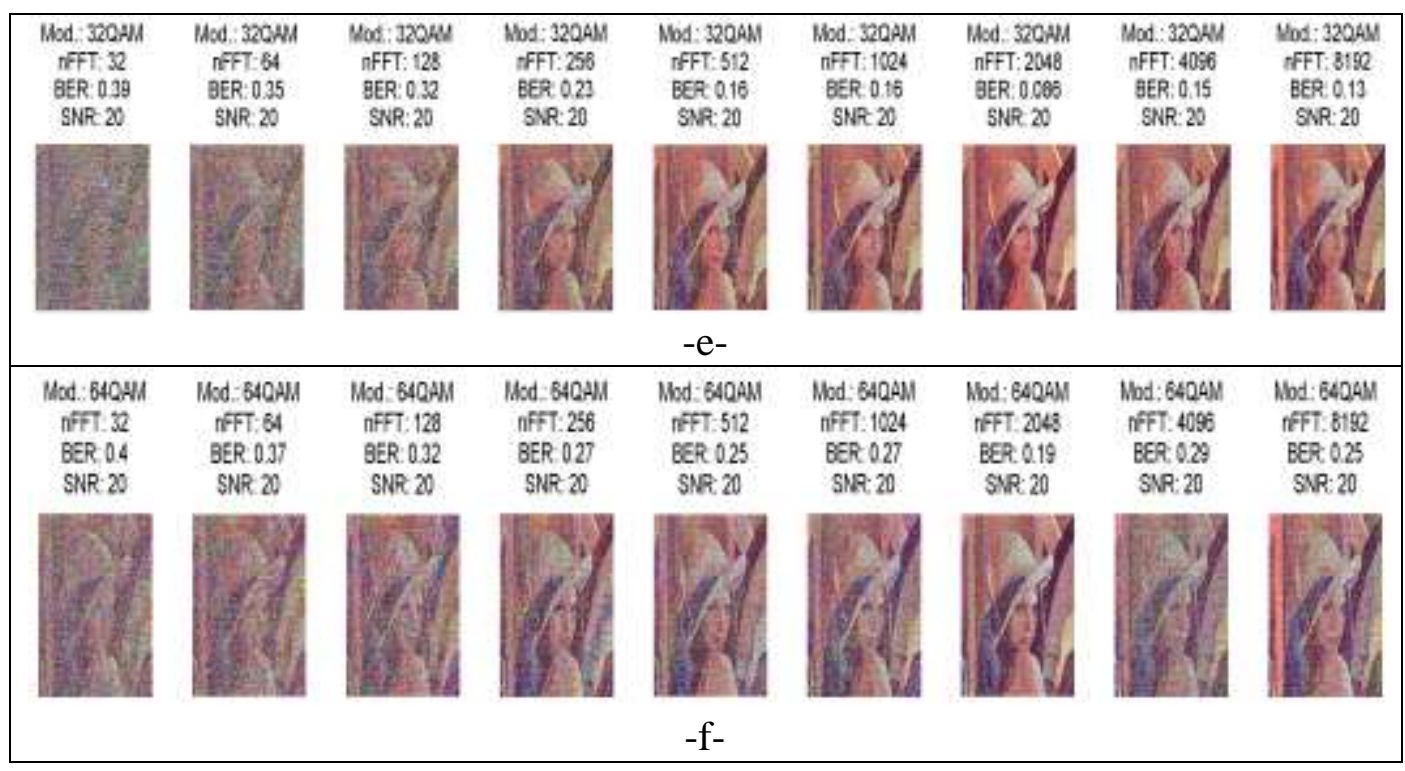

Figure 2. Receiving Image Using Different Type of Modulation, (a) BPSK, (b) QPSK, (c) 8PSK, (d) 16QAM, (e) 32QAM, and (f) 64QAM

Table 1. BER Values for Different Modulation Types, With Different nFFT Values When SNR=20

\begin{tabular}{|c|c|c|c|c|c|c|}
\hline $\begin{array}{c}\text { Mod. } \\
\text { FFT }\end{array}$ & BPSK & QPSK & 8PSK & 16QAM & 32QAM & 64QAM \\
\hline $\mathbf{3 2}$ & 0.006 & 0.15 & 0.29 & 0.32 & 0.39 & 0.40 \\
\hline $\mathbf{6 4}$ & 0.0016 & 0.067 & 0.19 & 0.30 & 0.36 & 0.37 \\
\hline $\mathbf{1 2 8}$ & 0.0002 & 0.047 & 0.15 & 0.28 & 0.32 & 0.32 \\
\hline $\mathbf{2 5 6}$ & $4.2 \mathrm{e}-05$ & 0.013 & 0.087 & 0.24 & 0.23 & 0.27 \\
\hline $\mathbf{5 1 2}$ & $1.6 \mathrm{e}-06$ & 0.032 & 0.03 & 0.16 & 0.16 & 0.26 \\
\hline $\mathbf{1 0 2 4}$ & 0 & 0.00029 & 0.027 & 0.16 & 0.16 & 0.27 \\
\hline $\mathbf{2 0 4 8}$ & 0 & 0.0014 & 0.0052 & 0.12 & 0.086 & 0.19 \\
\hline $\mathbf{4 0 9 6}$ & 0 & $6.9 \mathrm{e}-05$ & 0.0034 & 0.12 & 0.15 & 0.29 \\
\hline $\mathbf{8 1 9 2}$ & 0 & 0.00049 & 0.00013 & 0.13 & 0.13 & 0.25 \\
\hline
\end{tabular}

\subsection{BER-SNR}

To apply the proposed algorithm of OFDM to the data were calculated as a binary form from stored image and using several types of modulation to calculate the value of BER in relation to the total of SNR values $(-5,0,5,10, \ldots ., 40)$ with making the value of nFFT constants for each case $(64,128,256,512,1024$, and 2048). The study was done in two sets of modulation, one set for phase shift keying (BPSK, QPSK, and 8PSK), and another for quadrature amplitude modulation (16QAM, 32QAM, and 64QAM). Each set contains three types of modulation; obviously each element in the two sets has different effects in the transmitted signal figure 3 . 

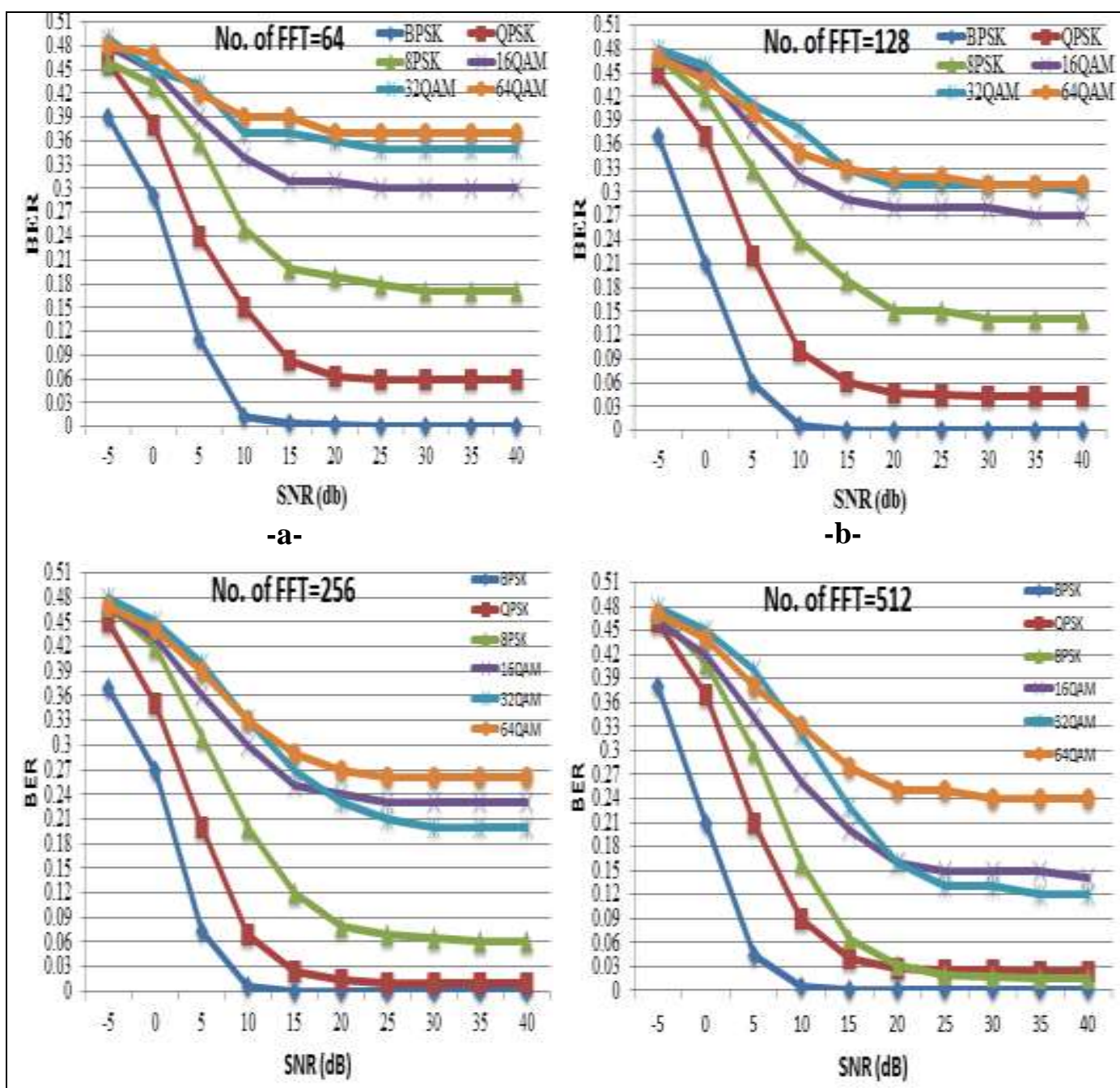

-c-
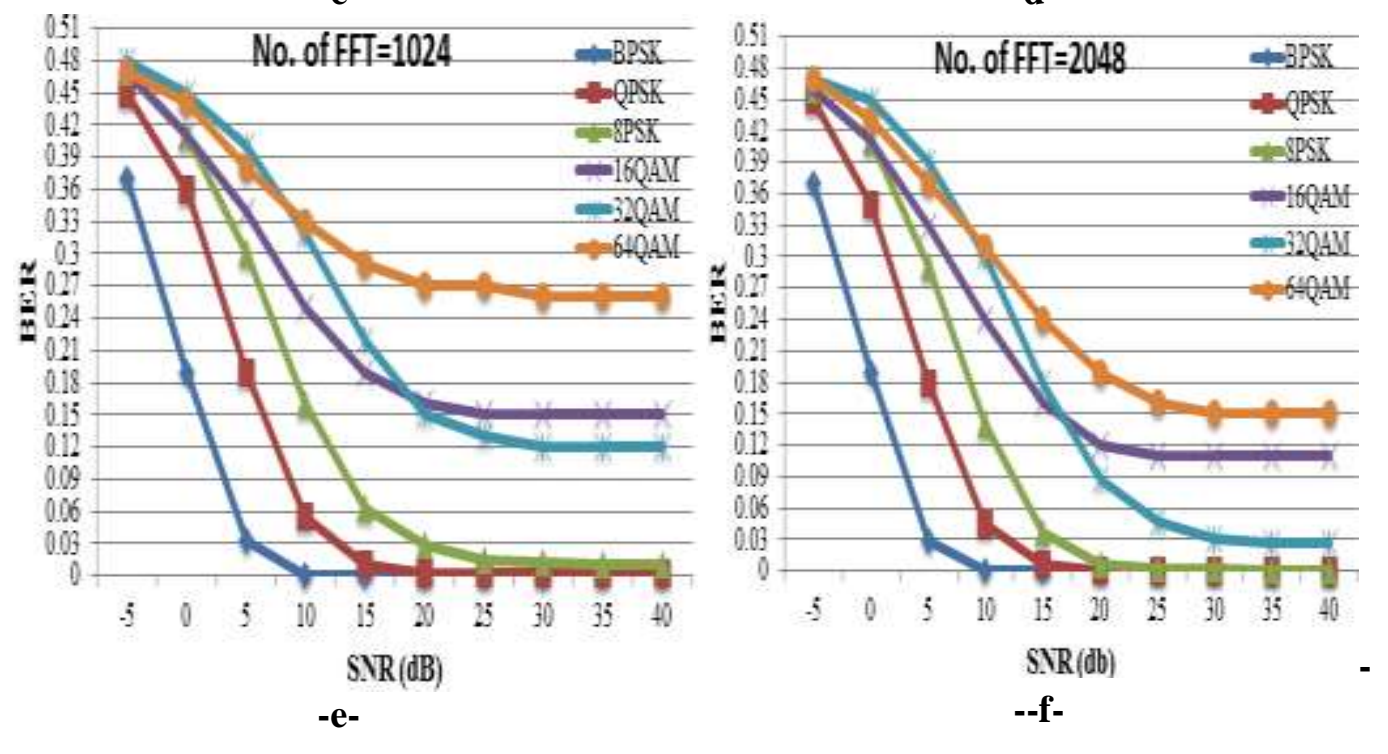

Figure 3. Performance of BER SNR With Different Size of FFT, (a) $\mathrm{nFFT}=64$, (b ) $\mathrm{nFFT}=128$, (c) $\mathrm{nFFT}=256$, (d) $\mathrm{nFFT}=512$, (e) $\mathrm{nFFT}=1024$, (f) $\mathrm{nFFT}=2048$ 
From the results of figures 2 and 3, the best modulation type used to transmit and receive an image is BPSK, especially when the SNR $=20 \mathrm{~dB}$ and higher, depending on the implementation of the algorithm and multi-values of SNR. Increased SNR value due to increased signal power that leads to a decrease in BER. Another choice will be QPSK then 8PSK. Thus, the BPSK is most robust over all types of PSK modulation because the highest level of noise is taken (or distorted), which causes the demodulation to make an incorrect choice. Symbol number of the FFT has no effect only if it is intended to improve the communication process. A Higher value of SNR leads to enhance the BER. Also, the modulation type which used in the operation, bandwidth, and system's noise are effects on the relation.

In this paper, the validation was compared with [25] to ensure our results. Authors in [25] take only four modulation type (BPSK, QPSK, 16QPSK, and QAM) for their comparison, while in this paper, six types of modulation had been taken. Also, they don't refer to the number of subcarriers or the size of the FFT and how its effects on the transmit and receive data. The result of BER SNR which obtained from this paper is better than in [25].

\subsection{BER-nFFT}

This part of the research results show the relation between BER and nFFT. Figure 4 reveals that the clearly visible thing of BPSK is the best modulation type, especially when $\mathrm{nFFT}=256$ and higher with SNR equal to $20 \mathrm{~dB}$ and more. Figure 4.d,e shows the curve of BPSK like a straight line, so at these values of SNR the FFT symbols have no effects. Therefore, the PSK is preferred over the QAM.

If we want to check the results when the SNR is less than zero, figure 4.a, clearly shows that the results are almost the same and reach near BER $=0$ when $\mathrm{nFFT}=131072$ and up. However, any increase in nFFT will increase the complexity (FFT multiplications) of calculation, which depends on equation 5 and as seen in Table 2.

$$
\text { Complexity }=(\mathrm{nFFT} / 2) \cdot \log 2(\mathrm{nFFT})
$$

Table 2. Relation Between nFFT and FFT Multiplication

\begin{tabular}{|c|c|c|c|c|c|c|c|c|}
\hline nFFT & 64 & 128 & 256 & 512 & 1024 & 2048 & 4096 & 8192 \\
\hline FFT Mul. & 192 & 448 & 1024 & 2304 & 5120 & 11264 & 24576 & 53248 \\
\hline
\end{tabular}

Furthermore, any increase in nFFT size will lead to increase the channel BW; for example, if $\mathrm{nFFT}=2048$ and suppose the time needed for calculating these nFFT equal to $100 \mu \mathrm{sec}$, then

Sampling frequency $(\mathrm{fs})=2048 / 100=20.48 \mathrm{MHz}, \mathrm{BW}=\mathrm{fs} / 2=10.24 \mathrm{MHz}$.

Table 3 shows relation between nFFT size and BW.

Table 3. Relation Between nFFT and BW

\begin{tabular}{|c|c|c|c|c|c|c|c|c|}
\hline nFFT & 64 & 128 & 256 & 512 & 1024 & 2048 & 4096 & 8192 \\
\hline BW (MHz) & 0.32 & 0.64 & 1.28 & 2.56 & 5.12 & 10.24 & 20.48 & 40.96 \\
\hline
\end{tabular}




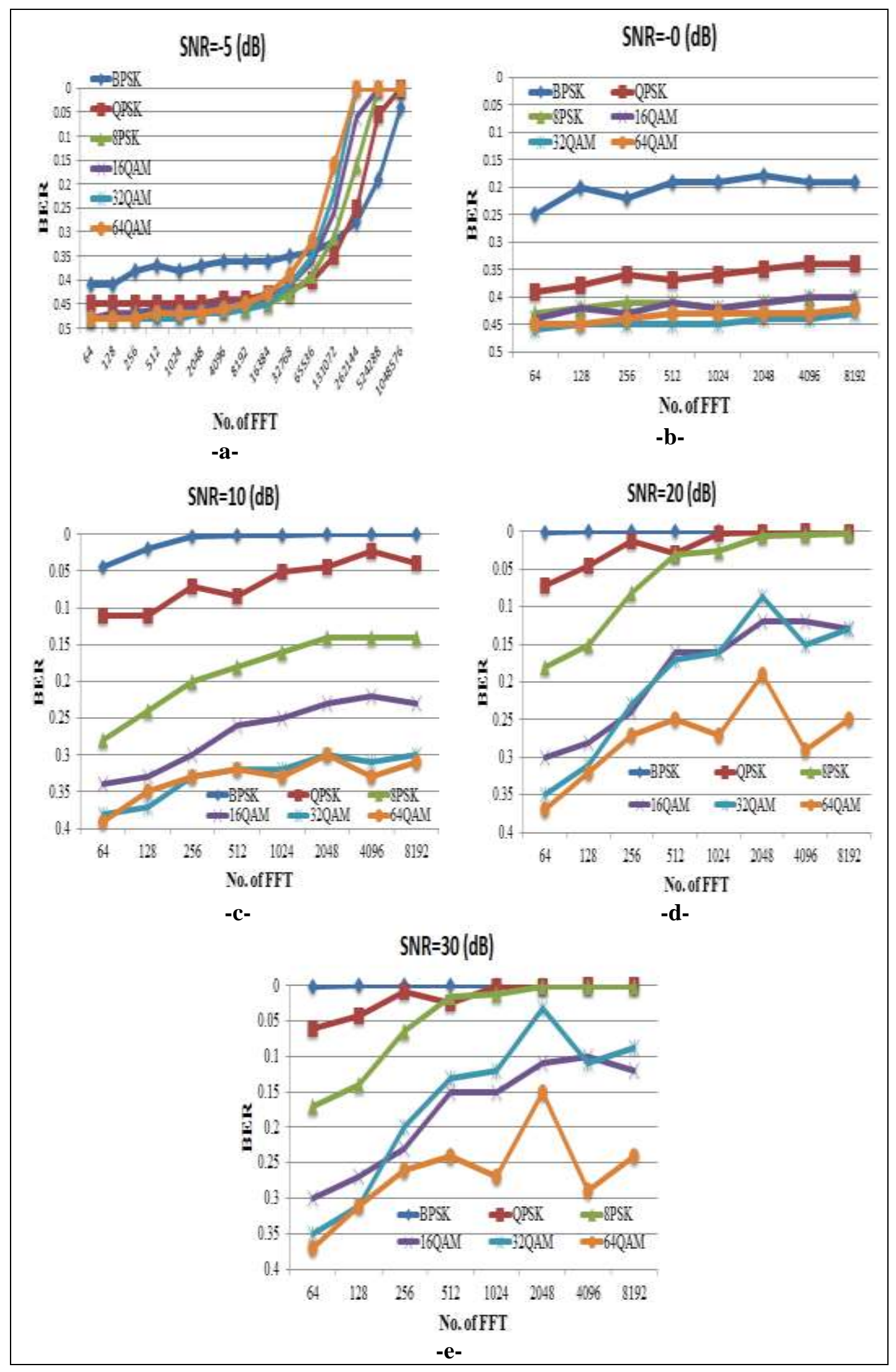

Figure 4. Relation Between BER nFFT With Different SNR, (a) SNR=-5, (b) SNR=0, (c) SNR=10, (d) SNR=20, and (e) $S N R=30$ 


\subsection{PAPR-nFFT}

From the figure 5 which is the relation between nFFT and PAPR, it is clear that this relation does not depend on SNR. Thus, in the event when the PAPR happened, how to find it, and how to minimize their effects on the signal should be discussed, then it is better and easier not to refer to the SNR value.

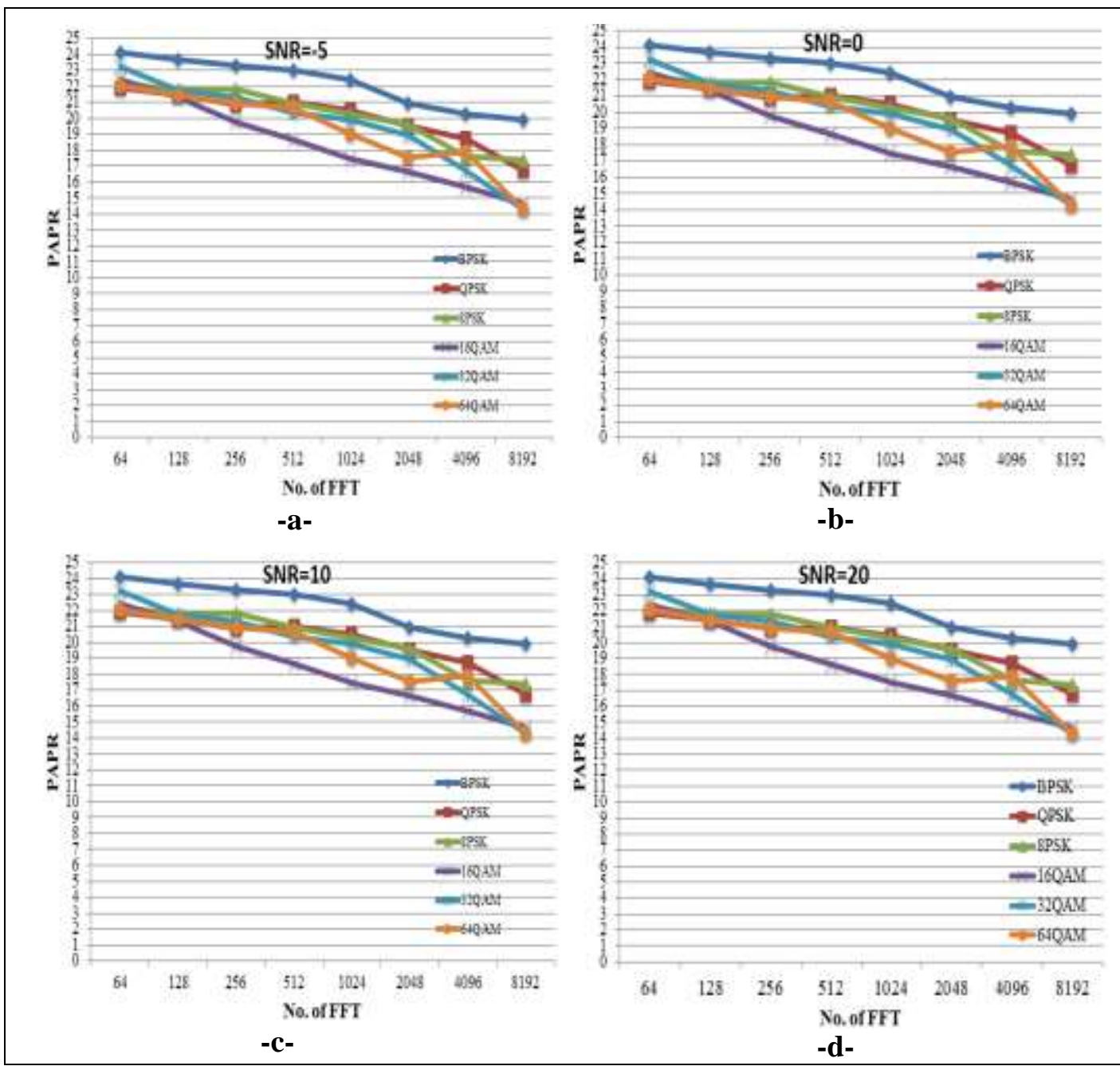

Figure 5. Relation Between PAPR nFFT With Different SNR, (a) $S N R=-5$, (b) $S N R=0$, (c) $S N R=10$, and (d) $S N R=20$

\section{Conclusion}

In this study, the OFDM structure was reviewed with a transceiver of the system. The algorithm had been done on this structure to send and receive an image in a noisy fading channel. Bit error rate is the most important performance of the OFDM system. Thus, the discussion and simulation were done on BER, especially with the several sizes of FFT and multi-values of SNR. The type of modulation and its control on the transmission and reception are considered. One important drawback of the system is the PAPR. Numerous methods are used to minimize the PAPR, but the easiest method is clipping and filtering. The major drawback is that in the case of increment the number of subcarriers, the quantity of the system will be significantly discredited and the efficiency of the spectrum usage will be decreased. 
If an image is transferred from one station to the other, the best modulation type is BPSK followed by QPSK and 8PSK, and this is obvious from the simulation results. Therefore, the set of phase shift keying is preferred over that of the set of QAM. The size number of FFT is also important, especially when they are increasing, but with a care of the bandwidth of the signal. Therefore, a balance must be established between the values (performances) to obtain the best image transmission from the sender to the recipient.

\section{References}

[1] A. F. Molisch, "Wireless Communications", Second Edition, University of Southern California, USA, John Wiley \& Sons Ltd, (2011).

[2] A. Agarwall and K. Agarwal, "Implementation and Performance Evaluation of OFDM System in Diverse Transmission Channel Using Simulink", American Journal of Electrical and Electronic Engineering, vol. 3, no. 5, (2015), pp. 117-123.

[3] V. Yatnalli and K. L. Sudha, "Image Transmission Over Multipath Fading Channel and Image Denoising Using Directional Weighted Median Filter", International Journal of Computer Applications, vol. 109, no. 10, (2015), pp.18-23.

[4] M. M. Salah and A. A. Elrahman, "Coded OFDM Scheme for Image Transmission Over Time-Varying Multipath Rayleigh Fading Channels", 15th IEEE Mediterranean Electro technical Conference, (2010), 602-607.

[5] R. Baranwal, "Optimization of PAPR Using HPA and Amplitude Clipping Reduction Technique", International Journal of Innovative Research in Computer and Communication Engineering, vol. 2, no. 4, (2014), pp. 3788-3793.

[6] V. S. Devi and S. V. Naresh, "An Effective Approach for Color Image Transmission Over OFDM for Video Broadcasting Applications", International Journal \& Magazine of Engineering, Technology, Management and Research, vol. 4, no. 12, (2014), pp. 26-31.

[7] D. Patne and P. N. Pusdekar, "Optimized Transmission of Images With OFDM Over AWGN Channel Using Trigonometric Transforms", International Journal of Science and Research (IJSR), vol. 5, no. 4, (2016), pp. 1540-1543.

[8] A. Sharma, S. De, and H. M. Gupta, "Energy-Efficient Transmission of DWT Image Over OFDM Fading Channel", Third International Conference on Communication System and Networks (COMSNETS), IEEE, (2011), pp. 1-7.

[9] M. N. A. Shareef and M. I. A. Ahsan, "Energy Efficient Transmission of DWT Image Over OFDM Using BPSK, QPSK and 16PSK", International Journal of Advanced Research in Computer Engineering \& Technology (IJARCET), vol. 4, no. 5, (2015), pp. 2444-2449.

[10] M. Elsharief, A. Zekry and M. Abouelatta, "Implementing a Standard DVB-T System Using MATLAB Simulink", International Journal of Computer Applications, vol. 98, no. 5, (2014), pp. 27-32.

[11] P. Dhok and A. Dhanvijay, "A Review on Digital Video Broadcasting Terrestrial (DVB-T) Based OFDM System", International Journal of Engineering and Techniques, vol. 1, no. 2, (2015), pp.27-31.

[12] L. P. Dachuri and N. Uppala, "Energy Efficient Transmission of Image Over DWT-OFDM System", International Journal of Electrical, Computer, Energetic, Electronic and Communication Engineering. vol. 7, no. 9, (2013), pp. 1264-1270.

[13] A. Soni and A. C. Tiwari, "Performance Analysis of OFDM System Under FFT, DWT and DCT Based Transform Techniques", International Journal of Emerging Technology and Advanced Engineering, vol. 4, no. 7, (2014), pp. 702-709.

[14] D. Li, X. Xiong and H. Wang, "Performance Analysis of CFO Estimation for OFDM Systems With Low-Precision Quantization", Wireless Communications, Networking and Applications, Springer India, 348, (2016), pp. 1005-1016.

[15] S. Sharma1 and J. Gupta, "Performance Analysis of OFDM With QPSK Using AWGN and Rayleigh Fading Channel", International Journal of Advances in Engineering \& Technology, vol. 6, no. 6, (2014), pp. 2635-2645.

[16] D. Jing and W. Yanmei, "Study on OFDM Symbol Timing Synchronization Algorithm", International Journal of Future Generation Communication and Networking, vol. 7, no. 1, (2014), pp. 43-50.

[17] B. R. Rao, K. Srivani and D. S. Prasanna, "ICI Cancellation Schemes and Selection Criteria in Orthogonal Frequency Division Multiplexing System: A Review”, International Journal of Engineering and Advanced Technology (IJEAT), vol. 2, no. 3, (2013), pp. 628-631.

[18] H. D. Joshi, "Performance Augmentation of OFDM System", PhD Thesis, Department of Electronics \& Communication Engineering, Jaypee University of Engineering \& Technology, India, (2012) May.

[19] S. Manzoor, V. Jeoti, N, Kamel and M. Asif Khan, "Novel SNR Estimation Technique In Wireless OFDM Systems", International Journal of Future Generation Communication and Networking, vol. 4, no. 4, (2011), pp. 1-20.

[20] S. Sengar and P. P. Bhattacharya, "Performance Improvement in OFDM System by PAPR Reduction", Signal \& Image Processing : An International Journal (SIPIJ), vol. 3, no. 2, (2012), pp. 157-169. 
[21] S. Sharma and P. K. Gaur, "Survey on PAPR Reduction Techniques in OFDM System", International Journal of Advanced Research in Computer and Communication Engineering, vol. 4, no. 6, (2015), pp. 271-274.

[22] M. Bisht and A. Joshi, "Various Techniques to Reduce PAPR in OFDM Systems: A Survey", International Journal of Signal Processing, Image Processing and Pattern Recognition, vol. 8, no. 11, (2015), pp. 195-206.

[23] A. Sroy, R. Li, F. Zeng and M. Fall, "A Novel Iterative Clipping and Filtering Technique for PAPR Reduction of OFDM Signals: System Using DCT/IDCT Transform”, International Journal of Future Generation Communication and Networking, vol. 6, no. 1, (2013), pp. 1-8.

[24] J. Lim, "Is BER The Bit Error Ratio or The Bit Error Rate?", EDN Network, vol. 14, (2010).

[25] C. U. Ndujiuba, O. Oni and A. E. Ibhaze, "Comparative Analysis of Digital Modulation Techniques in LTE 4G Systems", Journal of Wireless Networking and Communications, vol. 5, no. 2, (2015), pp. 60-66 
International Journal of Future Generation Communication and Networking Vol.10, No.8 (2017) 